ISSN: 0212-5374

DOI: http://dx.doi.org/10.14201/et2014331121142

\title{
EL PROFESORADO DE EDUCACIÓN INFANTIL ANTE EL DESARROLLO SOSTENIBLE. NECESIDADES FORMATIVAS
}

\author{
Childhood Education Teachers towards Sustainable \\ Development. Training needs
}

\author{
Les enseignants de maternelle au développement durable. \\ Les besoins en formation \\ F. Eduardo García-EsteBAN* y M. ${ }^{\mathrm{a}}$ Ángeles Murga-MenOYO*** \\ * Licenciado en Fía y CC. de la Educación. Facultad de Educación. Universidad \\ de Zaragoza. 75009 Zaragoza. Correo-e: fgarciae@unizar.es \\ * Doctora en Filosofía y Letras (Sección cC. de la Educación). Teoría de la \\ Educación y Pedagogía Social de la UNED. 28040 Madrid. Correo-e: mmurga@ \\ edu.uned.es
}

Recibido: 18-11-2013; Aceptado: 20-04-2014; Publicado: 30-04-2015

BIBLID [0212-5374 (2015) 33, 1; 121-142]

Ref. Bibl. F. EDUARDO GARCÍA-ESTEBAN y M. ${ }^{a}$ ÁNGELES MURGA-MENOYO. El profesorado de educación infantil ante el desarrollo sostenible. Necesidades formativas. Enseñanza \& Teaching, 33, 1-2015, 121-142.

RESUMEN: En este artículo se aborda la figura del docente de educación infantil en el marco de la educación para el desarrollo sostenible. La investigación, un estudio descriptivo de carácter exploratorio, tiene dos principales objetivos: evaluar los conocimientos básicos que los maestros y maestras de dicho nivel educativo tienen sobre la educación para el desarrollo sostenible; y, complementariamente, explorar las afinidades que pudieran existir entre su actual práctica docente y la que exige el modelo de la Década de Naciones Unidas por la Educación para el Desarrollo Sostenible en tres aspectos significativos: principios, valores y procedimientos, utilizando 
como fuente de datos el punto de vista de los propios docentes. Se han empleado dos instrumentos de recogida de datos: una prueba objetiva de elección múltiple y un cuestionario, ambos elaborados ad hoc. Los resultados muestran la necesidad de reforzar la formación del profesorado de educación infantil para la adquisición de competencias en sostenibilidad.

Palabras clave: formación del profesorado; competencias en sostenibilidad; educación para el desarrollo sostenible; educación infantil; práctica docente.

SUMMARY: This paper addresses the figure of teachers of early childhood education in the context of education for sustainable development. The investigation, a descriptive exploratory study has two main objectives: to assess the basic knowledge that the teachers of this educational level have on education for sustainable development and additionally, to explore the similarities that may exist between the current teachers teaching practice and the one required by the United Nations Decade of Education for Sustainable Development model in three significant ways: principles, values and procedures, using the teacher's perspective as data source. We have employed two data collection instruments: an objective test of multiple choices and a questionnaire, both made ad hoc. The results show the need to strengthen the training of childhood education teachers to acquire competences in sustainability.

Key words: teacher-training; competences in sustainability; education for sustainable development; childhood education; teaching practice.

RÉSUMÉ: Cet article aborde le professeur d'éducation de la petite enfance dans le cadre de l'éducation pour le développement durable. L'enquête, une étude exploratoire descriptive a deux objectifs principaux: évaluer les connaissances de base que les enseignants de ce niveau d'enseignement ont sur l'éducation pour le développement durable et d'explorer les similitudes qui peuvent exister entre les pratiques actuelles d'enseignement des enseignants et les requis du modèle Décennie des Nations Unies pour l'éducation au développement durable sur trois aspects importants: les principes, les valeurs et les procédures, en utilisant comme source de données au niveau des enseignants eux-mêmes. Nous avons utilisé deux instruments de collecte de données: un test objectif à choix multiple et un questionnaire. Les résultats montrent la nécessité de renforcer la formation des enseignants de la maternelle à acquérir des compétences en matière de durabilité.

Mots clé: formation des enseignants; compétences en matière de durabilité; éducation pour le développement durable; éducation de la petite enfance; enseignement pratique.

\section{INTRODUCCIÓN}

Un desafío mundial que las actuales sociedades tienen planteado, reconocido desde hace años por instituciones y organismos internacionales (Comisión Mundial 
del Medio Ambiente y del Desarrollo, 1988; Organización de las Naciones Unidas, 1972, 1992, 2002 y 2012), es avanzar hacia un desarrollo sostenible. El cambio social que exige no es menor sino paradigmático; conlleva una profunda transformación de los estilos de vida occidentales hasta consolidar principios y valores hoy descuidados, cuando no relegados al olvido. Porque, aun cuando el concepto de desarrollo sostenible permita interpretaciones diversas, existe un denominador común irrenunciable cualquiera que sea el enfoque adoptado. La Unesco lo recuerda cuando afirma:

A lo largo de su historia, la Organización de las Naciones Unidas ha defendido los valores relacionados con la dignidad humana, las libertades fundamentales, los derechos humanos, la equidad y el cuidado del medio ambiente. El desarrollo sostenible lleva esos valores un paso adelante, ampliándolos más allá de la generación actual a las generaciones futuras. Desarrollo sostenible significa valorar la biodiversidad y la conservación, junto con la diversidad humana, la inclusión y la participación. En el ámbito económico, hay quienes defienden la satisfacción de las necesidades para todos, mientras que otros prefieren la igualdad de oportunidades económicas.

Y añade que la Carta de la Tierra es una "Declaración de principios éticos fundamentales para construir una sociedad mundial justa, sostenible y pacífica" (Unesco, 2012b: 6-7).

En diciembre de 2002, las Naciones Unidas declararon la Década por la Educación para el Desarrollo Sostenible (2005-2014) -UNDESD- haciendo un llamamiento internacional para reorientar la educación y la formación hacia este nuevo modelo sociocultural. Con ello, una vez más, quedaba reconocida la función transformadora de la educación; y se reclamaba su papel como motor de un cambio que, extendido a todas las esferas de la sociedad global, pudiera propiciar una cultura de la sostenibilidad. Así queda recogido en el documento difundido al respecto por la Unesco (2006).

En plena sintonía con estos planteamientos, el Consejo de la Unión Europea celebrado a finales de 2010 reconocía que «según sus enseñanzas habituales, los profesores y formadores de todos los niveles de educación y formación harán frente a una serie de desafíos pedagógicos particulares a la hora de enseñar un tema transversal como la educación para el desarrollo sostenible, por lo que necesitarán una formación adecuada" (Consejo de la Unión Europea, 2010: 327/12). Y, a continuación, instaba a los Estados miembros a "proporcionar a los profesores, formadores, personal y directores de los centros la conciencia, el conocimiento y las competencias necesarias para promover e incluir los principios subyacentes a la educación para el desarrollo sostenible en sus planteamientos de la enseñanza y la gestión" (ibidem: 327/12).

Sin embargo, por mucho que los organismos internacionales lo promuevan $\mathrm{O}$, incluso, las directrices y normativas institucionales de los Estados lo permitan, del conocimiento que los profesores tengan sobre el desarrollo sostenible y del grado en que su práctica profesional cotidiana se ajuste a los principios y valores 
F. EDUARDO GARCÍA-ESTEBAN Y M. ${ }^{a}$ ÁNGELES MURGA-MENOYO

de este enfoque, de ambos factores, dependerá en buena medida el éxito en la implementación del modelo. Conocer la situación al respecto y ofrecer una base a líneas formativas optimizadoras a partir de las necesidades detectadas es el motivo que ha dado lugar a la investigación que presentamos a continuación. Se trata de un estudio descriptivo que busca constatar si los maestros y maestras de educación infantil tienen conocimientos básicos sobre la educación para el desarrollo sostenible y, complementariamente, explorar las afinidades que pudieran existir entre su actual práctica docente y aquella que la Unesco solicita en el marco de la Década (UNDESD). En la exposición del trabajo, en primer lugar, se describe el marco de la investigación: los antecedentes, el contexto y el perfil docente propio de una educación para el desarrollo sostenible, que sirve de referente. Las preguntas que se suscitan dan lugar a los objetivos de la investigación, el diseño metodológico y los resultados, seguidos de las correspondientes conclusiones y propuestas optimizadoras.

\section{MARCO DE LA INVESTIGACIÓN}

\subsection{Contexto y antecedentes}

En España, con la implantación de la ley Orgánica 1/1990 de 3 de octubre, de Ordenación General del Sistema Educativo (LOGSE), la educación ambiental -que es una educación para el desarrollo sostenible- se introdujo oficialmente en el sistema escolar. Se incorporó al currículo como un eje transversal, aunque en algunos centros también ha recibido, complementariamente, un tratamiento disciplinar en el nivel de la educación secundaria obligatoria (Murga-Menoyo y García del Pino, 2010). Una amplia bibliografía da cuenta de lo que fue sin duda una interesante innovación curricular que inició en la escuela un cambio de rumbo hacia la sostenibilidad (Gutiérrez-Pérez y Perales-Palacios, 2012; Uceda y Fort, 2011; Vega Marcote y Álvarez Suárez, 2006; Meinardi, Adúriz-Bravo y Revel, 2002; García, 2002; González Muñoz, 1994; Membiela y Pías, 1994).

Igualmente, en las décadas que han transcurrido desde entonces, la difusión de la Agenda 21 escolar y su implantación en los centros docentes ha sido creciente, contribuyendo a la sensibilización de la comunidad educativa, incluido el profesorado, en los principios y valores de la educación para el desarrollo sostenible (García Gómez y Verdugo Perona, 2012; Bautista-Cerro, 2011; Aznar Minguet, 2003).

Ambas líneas estratégicas, una en el terreno de la educación formal, otra, la segunda, en el marco de la educación no formal, permiten afirmar que el enfoque de la sostenibilidad, vehiculado a través de la educación ambiental, está presente desde hace años en nuestro contexto educativo. Sin duda habrá contribuido al despertar de los valores y actitudes característicos de este enfoque que diferentes estudios han detectado en la población y en grupos específicos (Cebrián y Junyent, 2014; Murga-Menoyo, 2008; Vázquez y Massanero, 2005; Berenguer y Corraliza, 2000), aunque faltan investigaciones al respecto en el nivel de la Educación Infantil. 
Por otra parte, con la promulgación de la LOGSE la Educación Infantil quedó integrada en nuestro sistema educativo, con entidad y normativa propia. Se articula en dos ciclos que la ley permite que puedan ser impartidos bien en un mismo centro educativo o en centros diferentes. En la Comunidad Autónoma de Aragón, contexto de nuestro estudio, en la mayoría de los casos se imparten en centros diferenciados, tanto por su ubicación física como por sus características y funcionamiento. Todos ellos están sujetos a la legislación estatal y a la normativa autonómica; esta última regula, entre otros aspectos, el currículo que ha de aplicarse como desarrollo de las enseñanzas mínimas que aquella determina a nivel estatal.

El Real Decreto 1333/1991, que establecía el currículo de Educación Infantil hasta la reciente promulgación de la actual ley de educación, mencionaba expresamente como objetivo de esta etapa: "observar y explorar el entorno inmediato con una actitud de curiosidad y cuidado, identificando las características y propiedades más significativas de los elementos que lo conforman y alguna de las relaciones que se establecen entre ellos" (at. 4e.). Texto que daba pie a distintas concreciones, entre otras las propias de una educación para el desarrollo sostenible.

En el caso de la Comunidad Autónoma de Aragón, la legislación vigente en los últimos diez años define un marco que permite implementar los planteamientos de la Década de Naciones Unidas por la Educación para el Desarrollo Sostenible; incluso, en algunos aspectos lo facilita explícitamente. Principios y valores como: la visión de conjunto (globalización), la importancia de lo local, el cuidado y respeto por el medio ambiente, la atención a la diversidad, la igualdad y equidad de género, centrales en el modelo educativo de la Década, se encuentran reflejados en la normativa. Sin embargo, se soslayan aspectos, igualmente esenciales para la sostenibilidad, como son: la interrelación global/local, la toma de decisiones en situaciones de incertidumbre, el funcionamiento interrelacionado de los sistemas naturales, sociales y económicos, o la prevención ambiental y el consumo responsable. Aspectos imprescindibles en el perfil docente propio de la educación para el desarrollo sostenible, como veremos a continuación

\subsection{Un perfil docente para una sociedad sostenible}

La primera gran cumbre mundial sobre desarrollo sostenible (Río 92) esboza como características necesarias de la educación para la sostenibilidad, entonces asumidas por la educación ambiental, las siguientes: a) contemplar interrelacionadas las diferentes dimensiones del medio ambiente: natural físico-biológica, socioeconómica y cultural; b) aceptar la sostenibilidad como eje transversal del currículo académico; c) utilizar junto a los tradicionales procedimientos formativos, otros innovadores y no formales; d) dirigirse a todos los colectivos y niveles, desde la edad escolar hasta la adulta (Organización de las Naciones Unidas, 1992: cap. 36.3).

Se trata de un modelo educativo que tiene en el aprendizaje activo y participativo una de sus señas de identidad. Los casos de buena práctica detectados en la revisión de la situación internacional -realizada por el grupo de expertos encargado por Unesco del seguimiento de la Década- indican que los profesores 
comprometidos con el modelo "animan a los educandos a realizar acciones como formular preguntas reflexivas y críticas, aclarar valores, plantearse futuros más positivos, pensar de modo sistémico, responder a través del aprendizaje aplicado, explorar la dialéctica entre tradición e innovación" (Tilbury, 2011: 31). E, igualmente, afirman los expertos que existen muestras de la reflexión crítica en todos los niveles educativos en los que se ha implementado el modelo, incluida la educación de la primera infancia (Davis, 2009; Elliot, 2010). Por tanto, cabe asegurar que las competencias que sustentan tales comportamientos se encuentran entre las que precisan los docentes para afrontar con éxito las metas del modelo. Y es necesaria una formación sistemática que facilite su adquisición.

En línea con esta necesidad, la Comisión Económica de Naciones Unidas para Europa (UNENCE) ha promovido una estrategia de apoyo a la educación por el desarrollo sostenible en cuyo marco se proponen un elenco de competencias básicas en sostenibilidad y unas directrices para su adquisición por los docentes. Ya en 2005 la Unesco había destacado como método innovador para integrar la sostenibilidad en la formación inicial y continua del profesorado "el modelo de competencias", según el cual todas las disciplinas y todos los docentes pueden contribuir a la educación para la sostenibilidad (Unesco, 2005: 74).

En el Informe de los expertos designados por unENCE (Comisión Económica de Naciones Unidas para Europa, 2012) se toman como referente los cuatro conocidos grandes pilares de la educación: aprender a aprender, aprender a hacer, aprender a ser y aprender a vivir juntos (Delors, 1994), y, entretejidos con ellos, tres ejes transversales: la dimensión holística, la anticipatoria y, una tercera, la transformadora. En los doce cuadrantes que resultan de las interrelaciones entre los siete elementos/ dimensiones se encajan, según queda reflejado en la Tabla 1, las competencias propias del perfil docente específicamente necesario para una sociedad sostenible.

Profesionales con ese perfil competencial podrán cumplir las funciones docentes propias del modelo que solicita la Unesco (2010: 97). Un modelo que requiere, como ya se anunciaba en los momentos iniciales de la Década (Unesco, 2005), transformar el desarrollo profesional de los facilitadores y la formación de los docentes; tesis que suscribe la Cumbre Rio+20, cuando, en el texto de la propia Declaración final, señala como prioridad la mejora de la capacidad de los sistemas educativos para contribuir al desarrollo sostenible y, entre las principales cuatro estrategias para lograrlo, incluye la de capacitar a los docentes facilitando su especialización en educación para el desarrollo sostenible (Organización de las Naciones Unidas, 2012: 49, punto 230).

La formación de los docentes, inicial y continua, en las competencias necesarias para que su práctica profesional se ajuste a las metas de la educación para el desarrollo sostenible es, pues, una tarea ineludible. Para abordarla es preciso partir de las necesidades de cada contexto particular. Y entre los factores que las condicionan se encuentran los conocimientos y las actitudes de los docentes, cuyo análisis, en nuestro contexto educativo y en el nivel de la Educación Infantil, apenas ha merecido atención. 
TABLA 1

Competencias del profesor en la educación para el desarrollo sostenible

\begin{tabular}{|c|c|c|c|}
\hline & DiMENSIÓN HOLÍSTICA & DIMENSIÓN ANTICIPADORA & $\begin{array}{c}\text { DIMENSIÓN } \\
\text { TRANSFORMADORA }\end{array}$ \\
\hline 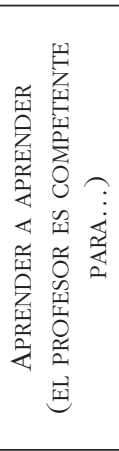 & $\begin{array}{l}\text { Integrar pensamiento y } \\
\text { práctica. } \\
\text { Apreciar el funcionamiento } \\
\text { interrelacionado de los } \\
\text { sistemas naturales, sociales } \\
\text { y económicos. } \\
\text { Comprender las } \\
\text { conexiones inter- e } \\
\text { intrageneracionales, } \\
\text { entre grupos culturales } \\
\text { y sociales, los seres } \\
\text { humanos y la naturaleza. }\end{array}$ & $\begin{array}{l}\text { Identificar la conexión } \\
\text { entre futuros sostenibles } \\
\text { y la manera en que } \\
\text { pensamos, vivimos y } \\
\text { trabajamos. } \\
\text { Cultivar la reflexión crítica } \\
\text { orientada a la mejora. } \\
\text { Afianzar el pensamiento } \\
\text { creativo y visionario en la } \\
\text { planificación del futuro. }\end{array}$ & $\begin{array}{l}\text { Transformar la manera } \\
\text { en que educamos y } \\
\text { aprendemos. } \\
\text { Preparar a los alumnos } \\
\text { para afrontar los } \\
\text { nuevos retos. } \\
\text { Aprovechar la } \\
\text { experiencia de los } \\
\text { alumnos como base } \\
\text { para la transformación. }\end{array}$ \\
\hline 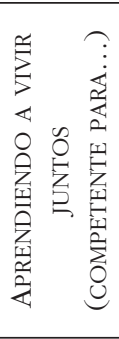 & $\begin{array}{l}\text { Implicar activamente a } \\
\text { los diferentes grupos a } \\
\text { través de las generaciones, } \\
\text { las culturas, lugares y } \\
\text { disciplinas. } \\
\text { Practicar el aprendizaje } \\
\text { colaborativo. }\end{array}$ & $\begin{array}{l}\text { Fomentar la negociación } \\
\text { de futuros alternativos } \\
\text { para un desarrollo } \\
\text { sostenible. } \\
\text { Trabajar con diferentes } \\
\text { perspectivas acerca de } \\
\text { dilemas, problemas, } \\
\text { tensiones y conflictos. }\end{array}$ & $\begin{array}{l}\text { Ayudar a los } \\
\text { estudiantes a aclarar } \\
\text { su propia y otras } \\
\text { cosmovisiones, } \\
\text { mediante el diálogo } \\
\text { argumentativo. } \\
\text { Reconocer y aceptar } \\
\text { los aciertos de los } \\
\text { enfoques alternativos. }\end{array}$ \\
\hline 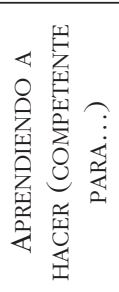 & $\begin{array}{l}\text { Compartir ideas y } \\
\text { experiencias en foros } \\
\text { interdisciplinares plurales. } \\
\text { Conectar al alumno con } \\
\text { sus ámbitos de influencia } \\
\text { local y global. }\end{array}$ & $\begin{array}{l}\text { Adoptar un enfoque } \\
\text { preventivo y precautorio. } \\
\text { Primar la evaluación } \\
\text { previa de las posibles } \\
\text { consecuencias de las } \\
\text { diferentes decisiones y } \\
\text { acciones. }\end{array}$ & $\begin{array}{l}\text { Aprovechar la } \\
\text { experiencia de los } \\
\text { alumnos como base } \\
\text { para la transformación. } \\
\text { Ejercitar la } \\
\text { participación activa y } \\
\text { comprometida. }\end{array}$ \\
\hline 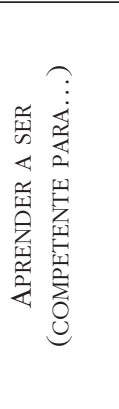 & $\begin{array}{l}\text { Tener en cuenta las } \\
\text { diferentes disciplinas, } \\
\text { culturas y puntos } \\
\text { de vista, incluidos } \\
\text { los conocimientos } \\
\text { y cosmovisiones de } \\
\text { contextos lejanos. } \\
\text { Facilitar la participación } \\
\text { activa de los estudiantes, } \\
\text { sin discriminaciones, en el } \\
\text { proceso de aprendizaje. }\end{array}$ & $\begin{array}{l}\text { Incentivar la contribución } \\
\text { positiva de los estudiantes } \\
\text { a otras personas y sus } \\
\text { entornos naturales y } \\
\text { sociales, a nivel local y } \\
\text { global. } \\
\text { Tomar decisiones } \\
\text { incluso en situaciones de } \\
\text { incertidumbre. }\end{array}$ & $\begin{array}{l}\text { Cuestionar los } \\
\text { supuestos subyacentes } \\
\text { a prácticas } \\
\text { insostenibles. } \\
\text { Ser un profesional } \\
\text { reflexivo (crítico e } \\
\text { innovador). } \\
\text { Involucrarse con los } \\
\text { alumnos para construir } \\
\text { relaciones positivas. }\end{array}$ \\
\hline
\end{tabular}

Fuente: Elaboración propia, basado en UNENCE (2012). 
F. EDUARDO GARCÍA-ESTEBAN Y M. ${ }^{a}$ ÁNGELES MURGA-MENOYO EL PROFESORADO DE EDUCACIÓN INFANTIL ANTE EL DESARROLLO SOSTENIBLE

Con este planteamiento, y como punto de partida de la investigación, se formularon las siguientes preguntas: ¿Poseen los maestros y maestras de Educación Infantil conocimientos básicos sobre la Década de Naciones Unidas por la Educación para el Desarrollo Sostenible (UNDEDS) y el modelo educativo que subyace? ¿Qué percepción tienen de su propia práctica docente en aspectos que pudieran reflejar principios y valores de dicho modelo? ¿Cabe pensar que su práctica docente es coherente con los principios y valores de la UNDEDS? ¿Cabe esperar que la actual práctica docente facilite la difusión ágil del modelo gracias a su coherencia con los principios y valores que aquella promueve? Preguntas que han dado paso a los objetivos de la investigación.

\section{OBjetivos}

En el marco contextual de la Comunidad Autónoma de Aragón, la investigación se ha centrado en el segundo ciclo de la Educación Infantil, con la finalidad última de fortalecer las posibilidades y paliar los obstáculos que esperan a los procesos de implementación del modelo que promueve la Década de Naciones Unidas por la Educación para el Desarrollo Sostenible (UNDEDS). Como objetivos concretos se establecieron dos principales:

- Realizar una evaluación de los conocimientos básicos que los maestros y maestras de dicho nivel educativo tienen sobre la educación para el desarrollo sostenible.

- Explorar las afinidades que pudieran existir entre la actual práctica docente de los maestros y maestras y la que exige el modelo UNDEDS, en tres aspectos significativos: principios, valores y procedimientos, utilizando como fuente de datos el punto de vista de los propios docentes.

Aunque se trata del caso particular de una única comunidad autónoma, con todas las salvedades que pudieran hacerse, consideramos que es un útil botón de muestra para una primera aproximación a la situación general en el contexto más amplio del Estado.

\section{POBLACIÓN Y MUESTRA}

La Comunidad Autónoma de Aragón cuenta con 385 centros de Educación Infantil y Primaria. El número de maestros y maestras titulados que ejercen en la especialidad alcanza los 1.500 sujetos.

Para determinar la muestra de la investigación se optó por utilizar un muestreo probabilístico y representativo de la población, atendiendo a los siguientes criterios: a) accesibilidad y disponibilidad mostrada por los centros para participar en el estudio; b) centros ubicados en capitales de provincia y en el medio rural; c) centros públicos y privados concertados. 
Se procedió a la aplicación de los instrumentos en 100 centros, teniendo en cuenta los criterios de representatividad indicados anteriormente. Se aplicó la siguiente fórmula de cálculo de muestra (Bugeda, 1974):

$$
\mathrm{n}=\frac{s^{2} \cdot p \cdot q \cdot N}{\mathrm{E}^{2} \cdot(\mathrm{N}-1)+s^{2} \cdot p \cdot q}
$$

Donde:

$n=$ es el número de elementos de la muestra que queremos obtener;

$s=$ es la desviación típica adecuada al nivel de confianza elegido;

$p=$ es el tanto por ciento de la característica estudiada;

$q=100-p$

En la mayoría de los estudios son: 50 para $p$ y 50 para $q$

$\mathrm{N}=$ es el tamaño de la población (poblaciones finales)

$\mathrm{E}=$ es el error de estimación que elegimos.

Teniendo en cuenta los criterios antes mencionados, donde $\mathrm{p}=\mathrm{q}=50$, un margen de error de $\pm 5 \%$ y un nivel de confianza del $99 \%$, se fija la cantidad de la muestra en 461 sujetos. Se han obtenido 499, por lo que se ha superado el nivel de confianza exigido.

Cabe destacar que un $84,4 \%$ de los profesores que han participado en la investigación son mujeres; el porcentaje superior de los sujetos, un 48\%, tiene más de 51 años; en un 80\% de los casos imparte docencia en centros públicos, y la mitad de ellos, un 50,3\%, habitan en poblaciones con más de 30.000 habitantes. Dada la concentración de los porcentajes, se estimó no pertinente la búsqueda de significatividad de las diferencias en función de las variables categóricas de la investigación.

\section{INSTRUMENTOS}

Con motivo de la investigación se diseñaron dos instrumentos de recogida de datos. Para detectar los conocimientos, una prueba objetiva. Y para recoger los datos sobre cómo perciben los docentes su práctica profesional, un cuestionario de autoevaluación. Ambos fueron sometidos al juicio de expertos, que avalaron su pertinencia (validez de contenido).

\subsection{Prueba objetiva de elección múltiple}

En la elaboración de la prueba objetiva sobre conocimientos se tuvieron en cuenta tanto aspectos teóricos como aspectos prácticos de la Década. Consta de veinte preguntas de elección múltiple, con cuatro opciones de respuesta -una de ellas válida- articuladas en dos bloques: a) información básica sobre la UNDEDS y b) metas de la educación para el desarrollo sostenible. El protocolo de la prueba estaba encabezado por preguntas destinadas a recoger los datos sociodemográficos de los participantes en la investigación: sexo, edad, tipo de centro y población donde se ubica el centro. 


\subsection{Cuestionario de autoevaluación}

El cuestionario de autoevaluación presenta, articulados en tres bloques, un total de 40 ítems. Los enunciados reflejan formas de hacer en la práctica del aula que los especialistas consideran relacionadas con los principios, valores y procedimientos de la educación para el desarrollo sostenible (Aznar y Ull, 2013; Junyent, Bonil y Caladell, 2011; Mogensen, Mayer, Breiting y Varga, 2009; Murga-Menoyo, 2013; Novo, 2006; Unesco, 2006, 1010, 2012a y 2012b). En su forma se ajusta a una escala tipo Likert con cinco opciones de respuesta, desde nada de acuerdo a totalmente de acuerdo, siendo el 1 la valoración inferior. Asimismo se contempla la opción de no sabe/no contesta.

Las pruebas de consistencia interna del cuestionario de autoevaluación arrojaron un 0,964 alfa de Cronbach, lo que significa un alto grado de fiabilidad. En la Tabla 2 se ofrecen los índices de homogeneidad de cada uno de los ítems. Únicamente en dos casos, el ítem 1 y el ítem 14, se encontraban por debajo del 0,2, por lo que su eliminación apenas hubiera supuesto el aumento de una milésima en el alfa global y, prevaleciendo un criterio práctico, se decidió mantener ambos ítems.

TABLA 2

Fiabilidad del cuestionario

\begin{tabular}{|c|c|c|c|c|}
\hline ÍtEM & $\begin{array}{c}\text { MEDIA DE LA ESCALA } \\
\text { SI SE ELIMINA EL } \\
\text { ELEMENTO }\end{array}$ & $\begin{array}{c}\text { VARIANZA DE LA } \\
\text { ESCLA SI SE ELIMINA } \\
\text { EL ELEMENTO }\end{array}$ & $\begin{array}{c}\text { CORRELACIÓN } \\
\text { ELEMENTO-TOTAL } \\
\text { CORREGIDA }\end{array}$ & $\begin{array}{c}\text { ALFA DE CRONBACH } \\
\text { SI SE ELIMINA EL } \\
\text { ELEMENTO }\end{array}$ \\
\hline 1 & 136,90 & 1123,631 &, 150 &, 965 \\
\hline 2 & 136,50 & 1069,953 &, 708 &, 962 \\
\hline 3 & 136,79 & 1072,063 &, 735 &, 963 \\
\hline 4 & 136,74 & 1068,355 &, 784 &, 962 \\
\hline 5 & 136,80 & 1097,069 &, 473 &, 964 \\
\hline 6 & 136,46 & 1076,458 &, 709 &, 963 \\
\hline 7 & 137,31 & 1071,851 &, 714 &, 963 \\
\hline 8 & 136,64 & 1098,889 &, 451 &, 964 \\
\hline 9 & 136,87 & 1085,428 &, 611 &, 963 \\
\hline 10 & 136,91 & 1094,707 &, 468 &, 964 \\
\hline 11 & 136,44 & 1065,761 &, 744 &, 963 \\
\hline 12 & 136,73 & 1087,075 &, 580 &, 963 \\
\hline 13 & 136,79 & 1077,083 &, 714 &, 965 \\
\hline 14 & 137,06 & 1123,719 &, 152 &, 962 \\
\hline 15 & 136,54 & 1070,940 &, 783 &, 965 \\
\hline 16 & 136,70 & 1107,025 &, 341 &, 962 \\
\hline 17 & 136,82 & 1065,298 &, 789 &, 964 \\
\hline 18 & 136,79 & 1092,837 &, 508 & \\
\hline
\end{tabular}




\begin{tabular}{|c|c|c|c|c|}
\hline ÍtEM & $\begin{array}{c}\text { MEDIA DE LA ESCALA } \\
\text { SI SE ELIMINA EL } \\
\text { ELEMENTO }\end{array}$ & $\begin{array}{c}\text { VARIANZA DE LA } \\
\text { ESCALA SI SE ELIMINA } \\
\text { EL ELEMENTO }\end{array}$ & $\begin{array}{c}\text { CORRELACIÓN } \\
\text { ELEMENTO-TOTAL } \\
\text { CORREGIDA }\end{array}$ & $\begin{array}{c}\text { ALFA DE CRONBACH } \\
\text { SI SE ELIMINA EL } \\
\text { ELEMENTO }\end{array}$ \\
\hline 19 & 136,66 & 1077,587 &, 686 &, 963 \\
\hline 20 & 136,77 & 1063,722 &, 736 &, 962 \\
\hline 21 & 136,74 & 1093,936 &, 494 &, 964 \\
\hline 22 & 136,52 & 1074,861 &, 721 &, 963 \\
\hline 23 & 136,79 & 1068,401 &, 796 &, 962 \\
\hline 24 & 136,51 & 1070,744 &, 762 &, 963 \\
\hline 25 & 136,61 & 1082,547 &, 620 &, 963 \\
\hline 26 & 136,81 & 1077,622 &, 702 &, 964 \\
\hline 27 & 136,77 & 1102,949 &, 390 &, 962 \\
\hline 28 & 136,51 & 1071,467 &, 772 &, 962 \\
\hline 29 & 136,56 & 1072,284 &, 776 &, 962 \\
\hline 30 & 136,75 & 1071,502 &, 763 &, 965 \\
\hline 31 & 136,71 & 1109,731 &, 318 &, 962 \\
\hline 32 & 136,47 & 1069,234 &, 702 &, 963 \\
\hline 33 & 136,73 & 1068,475 &, 697 &, 964 \\
\hline 34 & 136,84 & 1078,381 &, 688 &, 963 \\
\hline 35 & 136,87 & 1091,506 &, 522 &, 963 \\
\hline 36 & 136,82 & 1073,149 &, 711 &, 963 \\
\hline 37 & 136,65 & 1076,064 &, 706 &, 963 \\
\hline 38 & 136,71 & 1087,823 &, 583 &, 262 \\
\hline 39 & 136,87 & 1113,924 &, 585 & \\
\hline 40 & 136,93 & 1087,015 & & \\
\hline
\end{tabular}

\section{Procedimiento De RECOGIDA DE DATOS}

Se contactó telefónicamente con los coordinadores de ciclo de los centros seleccionados, se les explicó el propósito de la investigación y se solicitó su colaboración para la aplicación de los instrumentos. En los centros donde fue preciso contar con colaboradores externos para aplicar las pruebas, fueron entrenados e informados del propósito del estudio.

\section{Resultados}

\subsection{Nivel de conocimientos de los profesores}

La Figura 1 permite apreciar visualmente la situación. En primer lugar, cabe desatacar que el 83,6\% de los sujetos han superado la prueba de conocimientos, 
dato en principio satisfactorio aunque sin olvidar que los conocimientos evaluados son básicos y que el porcentaje mayor de los sujetos de este grupo, un 50,8\%, obtiene un mero aprobado.

FIGURA 1

Nivel de conocimientos básicos de los profesores

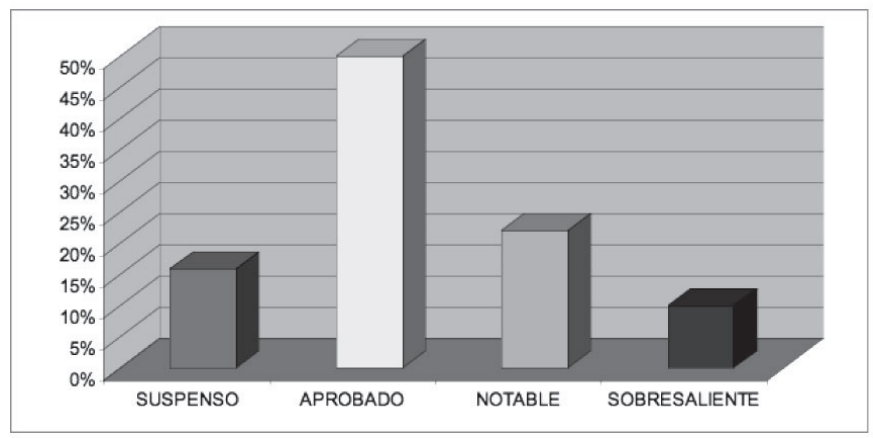

En el rango del notable se sitúa el 22,2\% de la muestra; y obtienen la calificación de sobresaliente el 10,6\% de los sujetos. Finalmente, un 16,4\% de los docentes no alcanza el nivel de aprobado. La media grupal se sitúa en el aprobado $(6,24)$, con una desviación típica de 1,72 que en la horquilla 1-5 de la escala no llama la atención.

\subsection{Autoevaluación de la propia práctica docente}

Si bien es preciso considerar las respuestas al cuestionario de autoevaluación en función de cada ítem, cabe afirmar que, contemplados los datos en su conjunto, la percepción que tienen los maestros y maestras sobre aspectos significativos de su práctica docente implícitamente relacionados con los objetivos, principios y valores de la educación para el desarrollo sostenible pone de manifiesto que, aun sin ser conscientes tal vez de ello, los docentes no están alejados del modelo educativo que promueve la UNDEDS.

Para facilitar la ordenación de los resultados se presentan sistematizados en dos principales bloques temáticos: principios y valores; con los procedimientos como eje transversal.

\subsubsection{Principios y sus correspondientes procedimientos de aula}

En la Tabla 3 se presentan los datos correspondientes a los principios que orientan la práctica educativa de los maestros y maestras y los correspondientes procedimientos de aula. Cabe destacar que un 57,1\% de los sujetos asegura proponerse en sus clases el logro de competencias transversales relacionadas con la 
educación para el desarrollo sostenible (ítem 1), mientras que una mayoría de los docentes considera que su práctica se inscribe, mucho o totalmente, en un paradigma formativo centrado en el alumno. Prueba de ello es que un $68 \%$ afirma promover en dicha medida el aprendizaje autónomo (item 15); un 45,7\% reconoce con el mismo énfasis adoptar el paradigma constructivista (ítem 14); un 46,7\% potenciar un aprendizaje reflexivo para la toma de decisiones (item 13); y un 57,3\% de los sujetos atender a la formación en los niños de un criterio propio frente a los llamamientos de la publicidad (ítem 12). Asimismo, el 54,5\% declara contextualizar los temas, aproximándolos al entorno del alumno (ítem 22).

TABLA 3

Principios y procedimientos de aula

\begin{tabular}{|c|c|c|c|c|c|c|c|c|}
\hline \multirow{2}{*}{ ÍTEM } & \multicolumn{6}{|c|}{ PORCENTAJES DE RESPUESTA } & \multirow[b]{2}{*}{ M } & \multirow{2}{*}{ D. T. } \\
\hline & 1 & 2 & 3 & 4 & 5 & $\mathrm{NS} / \mathrm{NC}$ & & \\
\hline $\begin{array}{l}\text { 1. Mi práctica docente incluye el } \\
\text { logro de competencias transversales } \\
\text { en los alumnos/as relacionadas con } \\
\text { la EDS. }\end{array}$ & 6,2 & 6,6 & 28,7 & 37,1 & 16 & 5,4 & 3,34 & 1,305 \\
\hline $\begin{array}{l}\text { 5. Siempre que puedo aplico el } \\
\text { método de Proyectos de Kilpatrick }\end{array}$ & 6,6 & 9,2 & 30,7 & 27,3 & 23,6 & 2,6 & 3,44 & 1,271 \\
\hline $\begin{array}{l}\text { 6. Todo el quehacer en mi clase } \\
\text { tiene en cuenta los centros de interés } \\
\text { de Decroly. }\end{array}$ & 4,4 & 10,6 & 23,2 & 18,2 & 41,9 & 1,6 & 3,78 & 1,297 \\
\hline $\begin{array}{l}\text { 8. Alterno actividades motrices que } \\
\text { requieren el predominio de diferentes } \\
\text { cualidades de peso, altura, etc. }\end{array}$ & 1,4 & 9 & 31,3 & 24 & 30,1 & 4,2 & 3,6 & 1,271 \\
\hline $\begin{array}{l}\text { 9. En el desarrollo de las actividades } \\
\text { físico-deportivas promuevo que } \\
\text { mis alumnos y alumnas conozcan y } \\
\text { practiquen diversos deportes, para } \\
\text { ello alterno el fútbol, baloncesto, } \\
\text { voleibol, etc. }\end{array}$ & 4 & 25,1 & 21,2 & 23 & 25,5 & 1,2 & 3,37 & 1,277 \\
\hline $\begin{array}{l}\text { 12. Busco sensibilizar al alumnado } \\
\text { para que cuando pidan que les } \\
\text { compren algo no se guíen por } \\
\text { la marca ni el precio sino por } \\
\text { sus gustos, que no tienen que } \\
\text { coincidir con los de la televisión } \\
\text { necesariamente. }\end{array}$ & 8,4 & 12,2 & 22 & 28,9 & 27,3 & 1,2 & 3,51 & 1,301 \\
\hline
\end{tabular}




\begin{tabular}{|c|c|c|c|c|c|c|c|c|}
\hline \multirow{2}{*}{ ÍTEM } & \multicolumn{6}{|c|}{ PORCENTAJES DE RESPUESTA } & \multirow{2}{*}{ M } & \multirow{2}{*}{ D. T. } \\
\hline & 1 & 2 & 3 & 4 & 5 & $\mathrm{NS} / \mathrm{NC}$ & & \\
\hline $\begin{array}{l}\text { 13. Pongo los medios para que antes } \\
\text { de tomar una decisión sobre alguna } \\
\text { cuestión, el alumnado analice los } \\
\text { pros y los contras. }\end{array}$ & 9,8 & 14,6 & 18,6 & 33,3 & 23,4 & 0,2 & 3,45 & 1,275 \\
\hline $\begin{array}{l}\text { 14. Considero el constructivismo } \\
\text { como instrumento básico en el } \\
\text { proceso de enseñanza aprendizaje en } \\
\text { las primeras etapas de la infancia. }\end{array}$ & 7 & 16 & 27,3 & 31,3 & 14,4 & 4 & 3,18 & 1,289 \\
\hline $\begin{array}{l}\text { 15. En clase utilizo la metodología } \\
\text { del aprendizaje por descubrimiento } \\
\text { autónomo. }\end{array}$ & 10,8 & 7 & 14 & 36,7 & 31,3 & 0,2 & 3,7 & 1,285 \\
\hline $\begin{array}{l}\text { 16. Utilizo los ordenadores del centro } \\
\text { o los Tablet PC para desarrollar } \\
\text { diferentes actividades con el } \\
\text { alumnado. }\end{array}$ & 2 & 14,8 & 30,9 & 15,8 & 33,3 & 3,2 & 3,54 & 1,311 \\
\hline $\begin{array}{l}\text { 18. Al iniciar un tema en clase } \\
\text { pregunto a mis alumnos y alumnas } \\
\text { sobre la cuestión a tratar. }\end{array}$ & 0,2 & 13 & 31,5 & 24 & 25,7 & 5,6 & 3,45 & 1,308 \\
\hline $\begin{array}{l}\text { 22. Contextualizo todos los aspectos } \\
\text { que trato en clase, utilizando } \\
\text { situaciones de mi localidad y } \\
\text { problemas conocidos por mi } \\
\text { alumnado. }\end{array}$ & 1,8 & 16,4 & 25,5 & 11,6 & 42,9 & 1,8 & 3,72 & 1,31 \\
\hline
\end{tabular}

M: media. D. T.: desviación típica. 1: Nada de acuerdo; 2: Algo de acuerdo; 3: Bastante de acuerdo; 4: Muy de acuerdo; 5: Totalmente de acuerdo. NS/C: No sabe/no contesta.

Respecto al enfoque metodológico de aula, la mayoría de los maestros y maestras que han participado en la investigación asegura primar el método de proyectos (ítem 5), con un 50,9\% de los docentes que se sitúan en la opción mucho o totalmente. Así mismo, un 60,1\% de los sujetos dicen organizar su docencia por centros de interés (ítem 6); un 49,7\% inicia la explicación de cada nuevo tema con un sondeo de los conocimientos de sus alumnos al respecto (ítem 18), e, igualmente, un 49,1\% declara haber incorporado las TIC a su práctica docente (ítem 16).

\subsubsection{Valores y sus correspondientes procedimientos de aula}

En cuanto a los valores que priman cotidianamente en las aulas describiremos los resultados agrupándolos en tres diferentes bloques. Los datos correspondientes a un primer bloque, solidaridad y ayuda mutua, aceptación de la diversidad cultural y respeto al otro, centrales para la sostenibilidad, se recogen en la Tabla 4. En prácticamente la totalidad de los ítems el porcentaje mayoritario de los docentes 
se sitúan en la opción mucho o totalmente. Así, por ejemplo, el respeto a la vida en sus distintas manifestaciones (item 24), la dignidad de las personas que ha de primar sobre los valores materiales (ítem 34) o la igualdad radical entre los seres humanos en su diversidad (ítem 10) obtienen porcentajes de respuesta del 69,8\%, el 56,1\% y 43,4\%, respectivamente.

TABLA 4

Valores I: solidaridad, respeto a la diversidad

\begin{tabular}{|c|c|c|c|c|c|c|c|c|}
\hline \multirow{2}{*}{ ÍTEM } & \multicolumn{6}{|c|}{ PORCENTAJES DE RESPUESTA } & \multirow{2}{*}{$\mathrm{M}$} & \multirow{2}{*}{ D. T. } \\
\hline & 1 & 2 & 3 & 4 & 5 & $\mathrm{NS} / \mathrm{NC}$ & & \\
\hline $\begin{array}{l}\text { 7. Siempre tengo presente en clase las } \\
\text { diferencias individuales del alumnado como } \\
\text { un elemento integrador y enriquecedor. }\end{array}$ & 5,2 & 6,4 & 38,3 & 31,7 & 6,6 & 11,8 & 2,93 & 1,384 \\
\hline $\begin{array}{l}\text { 10. Explico a los alumnos y alumnas } \\
\text { que todos somos iguales en dignidad } \\
\text { independientemente del lugar de donde } \\
\text { seamos o donde vivamos y de su género. }\end{array}$ & 0,2 & 9,8 & 38,7 & 20,4 & 23 & 7,8 & 3,33 & 1,353 \\
\hline $\begin{array}{l}\text { 21. Cada día inicio la clase con un saludo en } \\
\text { todas las lenguas que usan mis alumnos/as. }\end{array}$ & 1,2 & 15,4 & 21,4 & 31,9 & 25,3 & 4,8 & 3,5 & 1,31 \\
\hline $\begin{array}{l}\text { 23. Intento que los niños/as más aventajados/ } \\
\text { as ayuden al resto a la hora de colocarse la } \\
\text { ropa y atarse los cordones. }\end{array}$ & 15 & 1,6 & 29,5 & 28,9 & 24,6 & 0,4 & 3,45 & 1,314 \\
\hline $\begin{array}{l}\text { 24. En primavera, al ir al parque, les explico } \\
\text { la importancia de respetar todo tipo de vida. }\end{array}$ & 8,6 & 9,2 & 11 & 35,7 & 34,1 & 1,4 & 3,73 & 1,323 \\
\hline $\begin{array}{l}\text { 25. Destaco el trabajo que cada uno realiza y } \\
\text { lo pongo en valor como un aporte más a la } \\
\text { comunidad. }\end{array}$ & 5,8 & 12,8 & 22,2 & 22 & 35,3 & 1,8 & 3,63 & 1,328 \\
\hline $\begin{array}{l}\text { 32. Todos/as comparten las pinturas y demás } \\
\text { material fungible, independientemente de la } \\
\text { capacidad de aporte que tenga cada uno. }\end{array}$ & 4,4 & 10,4 & 32,7 & 9,2 & 42,9 & 0,4 & 3,77 & 1,289 \\
\hline $\begin{array}{l}\text { 33. Cuando a lo largo del curso se incorporan } \\
\text { al centro alumnos o alumnas, dedicamos el } \\
\text { primer día a hablar de su país o ciudad y les } \\
\text { convertimos en protagonistas del día. }\end{array}$ & 13 & 0 & 12 & 56,7 & 15 & 3,2 & 3,51 & 1,312 \\
\hline $\begin{array}{l}\text { 34. Explico a los alumnos que la riqueza no } \\
\text { reside en el dinero que tenga su familia o en } \\
\text { sus coches, sino en su valía personal y en su } \\
\text { calidad humana }\end{array}$ & 2,2 & 18,6 & 18,6 & 36,3 & 19,8 & 4,4 & 3,4 & 1,294 \\
\hline $\begin{array}{l}\text { 37. Explico a mi alumnado que el respeto a } \\
\text { los demás (padres, madres y compañeros/as) } \\
\text { es necesario para que ellos nos respeten a } \\
\text { nosotros }\end{array}$ & 5,2 & 10,8 & 14,4 & 40,5 & 25,5 & 3,6 & 3,59 & 1,31 \\
\hline $\begin{array}{l}\text { 38. Cuando a algún alumno/a se le olvida el } \\
\text { bocadillo invito a los demás a que compartan } \\
\text { con él su almuerzo. }\end{array}$ & 4 & 11,2 & 30,3 & 23 & 28,7 & 2,8 & 3,53 & 1,276 \\
\hline
\end{tabular}

M: media. D. T.: desviación típica. 1: Nada de acuerdo; 2: Algo de acuerdo; 3: Bastante de acuerdo; 4: Muy de acuerdo; 5: Totalmente de acuerdo. NS/C: No sabe/no contesta. 
F. EDUARDO GARCÍA-ESTEBAN Y M. ${ }^{a}$ ÁNGELES MURGA-MENOYO EL PROFESORADO DE EDUCACIÓN INFANTIL ANTE EL DESARROLLO SOSTENIBLE

A propósito de la promoción de la solidaridad y ayuda mutua, un 53,5\% de los docentes afirma inducir este tipo de conductas en situaciones concretas como atarse los zapatos o colocarse la ropa (ítem 23); incluso un 51,7\% anima a los niños a compartir la merienda (ítem 38), e, igualmente, un 52,1\% apoya en su aula el uso común de los materiales (ítem 32) y un un 57,3\% enfatiza los logros individuales como éxitos del grupo (ítem 25).

Otro valor que un alto porcentaje de maestros reconocen promover $\mathbf{m u}$ cho o totalmente es el respeto a padres y compañeros (ítem 37), un 66\% de los docentes, y la acogida de los diferentes que se incorporan al aula (ítem 33), un $79,9 \%$ de los docentes. Sin embargo, la mayoría no aprovecha suficientemente la diversidad del alumnado como elemento enriquecedor del aula; tan solo un $38,3 \%$ se autoevalúa en el rango de mucho o totalmente respecto a utilizar las diferencias como elemento integrador del aula (ítem 7). Quedan, por tanto, un $61,7 \%$ de los maestros y maestras que bien no se pronuncian, lo hacen tibiamente o aseguran que su práctica docente nunca incluye ese comportamiento, aunque cabe destacar que un $57,2 \%$ inicia el día con un saludo en todas las lenguas nativas de sus alumnos (ítem 21).

Un segundo bloque de valores, la participación y el diálogo, cuyos datos se muestran en la Tabla 5, también obtiene altos porcentajes de docentes en las opciones mucho y totalmente.

Por una parte, una mayoría de los maestros y maestras hacen del diálogo un elemento significativo de su práctica cotidiana. Un $73,6 \%$ asegura contar con este procedimiento para resolver los conflictos que surgen en el aula (ítem 11); incluso un 43,3\% afirma que suele pedir a los participantes en una disputa que expongan sus respectivas razones ante la clase (ítem 35); mientras que un 55,1\% de los sujetos lo hace para buscar una solución ante los problemas persistentes (ítem 36).

Por otra parte, cabe destacar, igualmente, que una mayoría de los encuestados pretende la participación activa de sus alumnos en la toma de decisiones. Así, en un $72,7 \%$ solicitan su opinión para elegir las actividades de aula (ítem 20) y un 47,1\%, para decidir el destino de las salidas extraescolares (ítem 19).

Un tercer bloque de ítems se centra en el cuidado de los recursos de la naturaleza y el valor de la eficiencia (o austeridad) de uso. Como se puede apreciar en la Tabla 6, considerando conjuntamente los porcentajes de respuesta en las opciones de la escala mucho o totalmente, se constata que de forma mayoritaria los docentes promueven en sus aulas la eficiencia en el uso racional de los recursos naturales como agua, electricidad o papel. 
TABLA 5

Valores II. Participación, diálogo

\begin{tabular}{|c|c|c|c|c|c|c|c|c|}
\hline \multirow{2}{*}{ ÍTEM } & \multicolumn{6}{|c|}{ PORCENTAJES DE RESPUESTA } & \multirow{2}{*}{ M } & \multirow{2}{*}{ D. T. } \\
\hline & 1 & 2 & 3 & 4 & 5 & $\mathrm{NS} / \mathrm{NC}$ & & \\
\hline $\begin{array}{l}\text { 11. En clase organizamos una "Asamblea de } \\
\text { Sabios/as" para solucionar mediante el diálogo } \\
\text { los problemas que se nos plantean. }\end{array}$ & 9,6 & 8,4 & 8 & 38,3 & 35,3 & 0,4 & 3,8 & 1,289 \\
\hline $\begin{array}{l}\text { 17. En las actividades lúdicas que llevamos } \\
\text { a cabo en el aula, participo de forma activa, } \\
\text { poniéndome siempre que es posible a la } \\
\text { "altura" del alumnado. }\end{array}$ & 11,8 & 1,6 & 23,8 & 37,5 & 21 & 4,2 & 3,42 & 1,384 \\
\hline $\begin{array}{l}\text { 19. En la realización de actividades extraescolares, } \\
\text { concretamente en las salidas, tengo en cuenta } \\
\text { la opinión de mis alumnos/as y les doy la } \\
\text { oportunidad de que ellos decidan entre varias } \\
\text { opciones. }\end{array}$ & 4,4 & 11,4 & 35,1 & 9,8 & 37,3 & 2 & 3,58 & 1,314 \\
\hline $\begin{array}{l}\text { 20. A la hora de diseñar actividades, además } \\
\text { de tener en cuenta los recursos de los que } \\
\text { dispongo y la PGA cuento con la opinión del } \\
\text { alumnado. }\end{array}$ & 14,2 & 0,2 & 9,4 & 59,1 & 13,6 & 3,4 & 3,47 & 1,339 \\
\hline $\begin{array}{l}\text { 35. Cuando hay alguna disputa entre el } \\
\text { alumnado invito a los implicados a que salgan } \\
\text { a la pizarra y expliquen las causas. }\end{array}$ & 1 & 24,8 & 28,1 & 13,8 & 29,5 & 2,8 & 3,37 & 1,312 \\
\hline $\begin{array}{l}\text { 36. Cuando un problema se repite de forma } \\
\text { reiterada, reúno a todos los niños y niñas en la } \\
\text { alfombra para buscar una solución conjunta. }\end{array}$ & 13,2 & 6,4 & 23,6 & 30,3 & 24,8 & 1,6 & 3,42 & 1,363 \\
\hline $\begin{array}{l}\text { 40. Inicio muchas sesiones de trabajo con un } \\
\text { vídeo didáctico y doy a mis alumnos y alumnas } \\
\text { la posibilidad de elegir entre las opciones } \\
\text { disponibles. }\end{array}$ & 4,8 & 12,4 & 30,9 & 28,5 & 19 & 4,4 & 3,31 & 1,29 \\
\hline
\end{tabular}

M: media. D. T.: desviación típica. 1: Nada de acuerdo; 2: Algo de acuerdo; 3: Bastante de acuerdo; 4: Muy de acuerdo; 5: Totalmente de acuerdo. NS/C: No sabe/no contesta.

TABLA 6

Valores III. Eficiencia en el uso de los recursos naturales

\begin{tabular}{|l|c|c|c|c|c|c|c|c|c|}
\hline \multicolumn{1}{|c|}{ ÍTEM } & \multicolumn{5}{|c|}{ PORCENTAJES DE RESPUESTA } & \multirow{2}{*}{ M } & \multirow{2}{*}{ D. T. } \\
\cline { 2 - 8 } & 1 & 2 & 3 & 4 & 5 & NS/NC & & \\
\hline $\begin{array}{l}\text { 2. Aprovecho los recortes de cartulina para } \\
\text { realizar una actividad posterior que previamente } \\
\text { he diseñado antes de utilizar una nueva. }\end{array}$ & 9,8 & 7,2 & 13,6 & 36,5 & 32,7 & 0,2 & 3,74 & 1,267 \\
\hline $\begin{array}{l}\text { 3. Únicamente renovamos el material cuando el } \\
\text { anterior ya se ha acabado. }\end{array}$ & 4,4 & 27,9 & 14,2 & 21 & 31,7 & 0,8 & 3,45 & 1,343 \\
\hline $\begin{array}{l}\text { 26. Todos los años participamos con mi } \\
\text { alumnado en la plantación de árboles o } \\
\text { plantas. }\end{array}$ & 12 & 12 & 14,6 & 41,3 & 19,6 & 0,4 & 3,43 & 1,285 \\
\hline
\end{tabular}


F. EDUARDO GARCÍA-ESTEBAN Y M. ${ }^{a}$ ÁNGELES MURGA-MENOYO EL PROFESORADO DE EDUCACIÓN INFANTIL ANTE EL DESARROLLO SOSTENIBLE

\begin{tabular}{|c|c|c|c|c|c|c|c|c|}
\hline \multirow{2}{*}{ ÍTEM } & \multicolumn{6}{|c|}{ PORCENTAJES DE RESPUESTA } & \multirow{2}{*}{ M } & \multirow{2}{*}{ D. T. } \\
\hline & 1 & 2 & 3 & 4 & 5 & $\mathrm{NS} / \mathrm{NC}$ & & \\
\hline $\begin{array}{l}\text { 27. Explico a mis alumnos/as la importancia del } \\
\text { reciclado. }\end{array}$ & 2 & 9,6 & 24,4 & 37,1 & 20,8 & 6 & 3,47 & 1,305 \\
\hline $\begin{array}{l}\text { 28. Cuando reparto folios para realizar alguna } \\
\text { actividad les recuerdo que sean prudentes en } \\
\text { su uso ya que cuantos más usen más árboles } \\
\text { hay que talar. }\end{array}$ & 11,2 & 4,8 & 14,4 & 36,3 & 32,9 & 0,4 & 3,73 & 1,293 \\
\hline $\begin{array}{l}\text { 29. Elaboramos entre todos/as un recipiente } \\
\text { donde colocamos el papel reciclado y les } \\
\text { informo de su uso posterior. }\end{array}$ & 0 & 17 & 28,1 & 13,2 & 39,5 & 2,2 & 3,69 & 1,27 \\
\hline $\begin{array}{l}\text { 30. Sólo utilizamos la luz eléctrica cuando es } \\
\text { estrictamente necesario. }\end{array}$ & 15,8 & 0,2 & 3,8 & 37,1 & 22,6 & 0,4 & 3,49 & 1,307 \\
\hline $\begin{array}{l}\text { 31. Cada día se encarga un alumno/a de revisar } \\
\text { que los grifos estén bien cerrados y todas las } \\
\text { luces apagadas al salir de clase. }\end{array}$ & 2,8 & 14 & 23,8 & 29,3 & 26,7 & 3,4 & 3,53 & 1,282 \\
\hline $\begin{array}{l}\text { 39. Solo dejo sacar punta a los colores cuando } \\
\text { realmente no tienen. }\end{array}$ & 5,2 & 11,8 & 25,3 & 33,7 & 19,6 & 4,4 & 3,37 & 1,307 \\
\hline
\end{tabular}

M: media. D. T.: desviación típica. 1: Nada de acuerdo; 2: Algo de acuerdo; 3: Bastante de acuerdo; 4: Muy de acuerdo;

5: Totalmente de acuerdo. NS/C: No sabe/no contesta.

Un 56\% de los maestros y maestras solicitan diariamente a un niño que se ocupe de que luces y grifos queden cerrados al terminar la jornada (item 31); un $69,2 \%$ optimiza el gasto de papel, sensibilizando a la vez a los niños sobre la necesidad de este hábito (ítem 28); un 53,3\% no deja sacar punta a los lápices sin necesidad (ítem 39); y un 59,7\% asegura utilizar la electricidad tan solo cuando es estrictamente necesario (ítem 30). Igualmente, un 52,7\% de los encuestados afirma que sólo renueva el material cuando el anterior se ha acabado (ítem 3), mientras que un $57,9 \%$ trata de sensibilizar a la clase en la importancia del reciclado (ítem 27).

Encontramos que la mayoría de los sujetos no solo promueven el uso eficiente de los recursos naturales, un 69,2\% optimizan el uso del papel (ítem 2) y un 52,7\% el reciclado (ítem 29), sino que incluso un 60,9\% participan directamente con sus alumnos en la plantación anual de árboles (26).

\section{CONCLUSIONES}

Los datos de la investigación permiten avanzar algunas conclusiones, si bien, por las características de la muestra, se impone la prudencia en la interpretación y generalización de los resultados.

Respecto a los conocimientos básicos de los maestros y maestras sobre la UNDEDS y el modelo educativo que subyace, resulta probado que la situación es manifiestamente mejorable. Un 16\% de los sujetos suspenden la prueba y otro 50\% de los docentes se sitúan en torno al suficiente; es decir, tienen unos conocimientos muy 
"justos" sobre el tema. Este hecho, junto a los resultados que arroja el cuestionario de autoevaluación de la práctica docente, hace intuir que al menos algunos maestros y maestras cuya práctica cotidiana podría ser considerada afin a los planteamientos de la Década no poseen sólidos conocimientos sobre el tema y actúan sin asociar con él su docencia, con posible pérdida de coherencia interna y, consecuentemente, de eficacia. Por tanto, es necesario hacer propuestas para dar a conocer la Década, su modelo educativo y los fines que este modelo pretende conseguir.

En cuanto a las conclusiones que se derivan de los resultados del cuestionario de autoevaluación, los datos avalan que la práctica docente cotidiana de la mayoría de los maestros y maestras de Educación Infantil, tal como ellos mismos se perciben y evalúan, mantiene una cierta coherencia con principios y valores significativos de la educación para el desarrollo sostenible. Sin embargo, se aprecian simultáneamente porcentajes, en algunos ítems incluso próximos al 30\% de los sujetos, que o bien no se pronuncian, o reconocen que su práctica docente no está de acuerdo, o lo está tan solo "algo", con dichos principios y valores.

Pero, además, aunque la actual práctica de la mayoría de los docentes no parece oponerse al modelo UNDEDS, tampoco cabe afirmar que facilite su consolidación pues falta la cohesión interna de un modelo educativo conscientemente articulado que pueda contribuir con eficiencia a las metas de la sostenibilidad.

La principal necesidad educativa que nos revelan las conclusiones de la investigación se refiere a las carencias formativas del profesorado, con importantes lagunas. Sin duda, para que los maestros y las maestras de Educación Infantil puedan implementar con eficacia el modelo en sus aulas han de mejorar sus competencias en sostenibilidad. Competencias que incluyen contenidos conceptuales, procedimentales y actitudinales, como conjunto de saberes no solo teóricos sino también prácticos que es preciso abordar tanto en la formación inicial como en la formación continua del profesorado.

En el primer caso, la reforma de las titulaciones universitarias propiciada por la construcción del Espacio Europeo de Educación Superior brinda una excelente ocasión para ello. Así lo considera la Conferencia de Rectores de las Universidades Españolas (2005) al recomendar incluir la formación de competencias en sostenibilidad en todas las titulaciones. Y, para este propósito, cabe aprovechar los mecanismos que dentro de cada universidad permiten revisar los proyectos docentes de las asignaturas, introducir cambios y subsanar carencias.

En el ámbito de la formación continua, los tres principales tipos de actividades hoy consolidados en esta modalidad: cursos, seminarios y grupos de trabajo, son, igualmente, una vía adecuada para facilitar a los docentes en ejercicio la posibilidad de conocer los planteamientos de la UNDEDS, tomar conciencia de su necesidad y aceptar el compromiso activo de ajustar su práctica docente a las exigencias del modelo.

Finalmente, como conclusión general de la investigación, cabe reafirmar la necesidad de intensificar la formación del profesorado en las competencias necesarias para liderar procesos de educación para el desarrollo sostenible, paradigma general adoptado por las Naciones Unidas (Unesco, 2012b). 


\section{REFERENCIAS BIBLIOGRÁFICAS}

Aznar, P. y Ull, M. ${ }^{a}$ A. (2013). La responsabilidad por un mundo sostenible. Propuesta educativa a padres y profesores. Bilbao: Desclée De Brouwer.

Aznar Minguet, P. (2003). Participación de las agencias educativas en el desarrollo sotenible a nivel local: hacia la Agenda 21 escolar. Revista Española de Pedagogía, 225, 223-242.

Bautista-Cerro, M. ${ }^{a}$ J. (2011). Exigencias de la A21E. Un aprendizaje basado en competencias. En M. Melendro Estefanía, M. ${ }^{a}$ Á. Murga-Menoyo y A. Cano Pérez. IDEAs. Iniciativas de Educación Ambiental para la Sostenibilidad (pp. 51-64). Madrid: UNED.

Berenguer, J. M. ${ }^{a}$ y Corraliza, J. A. (2000). Preocupación ambiental y comportamientos ecológicos. Psicothema, 12 (3), 325-329.

Bugeda, J. (1974). Manual de técnicas de investigación social. Madrid: Instituto de Estudios Políticos.

Cebrián, G. y Junyent, M. (2014). Competencias profesionales en Educación para la Sostenibilidad: un estudio exploratorio de la visión de futuros maestros. Enseñanza de las Ciencias, 32 (1), 29-49. http://dx.doi.org/10.5565/rev/ensciencias.877.

Comisión Económica de Naciones Unidas para Europa (2012). Learning for the future. Competences for Sustainable Development. Ginebra: United Nations Economic Commission for Europe (UNENCE).

Comisión Mundial del Medio Ambiente y del Desarrollo (CMMAD) (1988). Nuestro futuro común. Madrid: Alianza Editorial.

Conferencia de Rectores de las Universidades Españolas (2005). Directrices para la Sostenibilización Curricular. Documento aprobado por el Comité Ejecutivo del Grupo de Trabajo de Calidad Ambiental y Desarrollo Sostenible (CADEP) de la Conferencia de Rectores de las Universidades Españolas (CRUE), celebrado en Valladolid el 18 de abril de 2005. Descargado el 5 de septiembre de 2013. http://www.uah.es/universidad/ ecocampus/documentos/C3SostenibilizacionCurricular_CRUE.pdf.

Consejo de la Unión Europea (2010). Conclusiones del Consejo de 19 de noviembre de 2010 sobre la educación para el desarrollo sostenible (2010/C 327/05). Diario Oficial de la Unión Europea, pp. C 327/11- =C 327/14. http://eur-lex.europa.eu/LexUriServ/LexUriServ.do?uri=OJ:C:2010:327:0011:0014:ES:PDF.

Davis, J. (2009). Revealing the Research 'Hole' of Early Childhood Education for Sustainability: a Preliminary Survey of Literature. Environmental Education Research, 15 (2), 227-241. http://dx.doi.org/10.1080/13504620802710607

Delors, Jaques (1994). La educación encierra un tesoro. Madrid: Santillana/UnESCO.

Elliott, S. (2010). Essential, not Optional @ Education for Sustainability in Early Chilhood Centres. Education for Sustainability Echange Magazine, marzo-abril, 34-37.

García, J. E. (2002). Los problemas de la Educación Ambiental ¿es posible una Educación Ambiental integradora? Investigación en la Escuela, 46, 5-27.

García Gómez, J. y Verdugo Perona, J. (2012) La educación ambiental en la agenda 21 local de Sagunto (Valencia). Didáctica de las Ciencias Experimentales y Sociales, 26, 177197.

González Muñoz, M. ${ }^{a}$ C. (1994). Principales tendencias y modelos de la Educación Ambiental en el sistema escolar. Revista Iberoamericana de Educación, 11 (2), 13-74. 
Gutiérrez-Pérez, J. y Perales-Palacios, F. J. (2012). Ambientalización curricular y sostenibilidad. Nuevos retos de profesionalización docente. Revista de Curriculum y Formación de Profesorado, 16 (2), 5-14.

Junyent, M.; Bonil, J. y Caladell, G. ( 2011). Evaluar la ambientalización curricular de los estudios superiores: un análisis de la RED EDUSOT. Ensino Em Re-Vista, 18 (2), 323-340.

Ley Orgánica 8/2013, de 9 de diciembre, para la mejora de la calidad educativa. Boletín Oficial del Estado n. ${ }^{\circ} 295$ de 10 de diciembre de 2013.

Meinardi, E.; Adúriz-Bravo, A. y Revel, A. (2002). La Educación Ambiental en el aula. Una propuesta para integrar contenidos multidisciplinares a través de la argumentación. Investigación en la Escuela, 46, 93-103.

Membiela, P. y Pias, R. (1994). Problemática actual de la integración de la Educación Ambiental en el sistema educativo español. Revista Interuniversitaria de Formación del Profesorado, 21, 211-217.

Mongensen, F.; Mayer, M.; Breiting, S. y Narga, A. (2009). Educación para el desarrollo sostenible. Tendencias, divergencias y criterios de calidad. Barcelona: Graó.

Murga-Menoyo, M. ${ }^{a}$ Á. (2008). Percepciones, valores y actitudes ante el desarrollo sostenible. Detección de necesidades educativas en estudiantes universitarios. Revista Española de Pedagogía, 240, mayo-agosto, 327-343.

Murga-Menoyo, M. ${ }^{a}$ Á. (2013). Desarrollo Sostenible. Problemáticas, agentes y estrategias. Madrid: MacGraw-Hill.

Murga-Menoyo, M. ${ }^{a}$ Á. y García del Pino, I. (2010). La Carta de la Tierra y el blog. Dos elementos centrales de una experiencia docente innovadora en $4 .^{\circ}$ de EsO. Revista Enseñanza de las Ciencias, 28 (1), 85-94.

Novo, M. (2006). El desarrollo sostenible. Su dimensión ambiental y educativa. Madrid: Pearson.

ORGANIZACIÓN DE LAS NACIONES UnidAS (1972). Declaración final. Conferencia de las Naciones Unidas sobre el Medio Ambiente Humano, 5-10 de junio, Estocolmo. Descargado el 5 de septiembre de 2013.http://www.unep.org/Documents.Multilingual/Default.asp ?DocumentID=97\&ArticleID=1503\&l=en.

ORGANIZACIÓN DE LAS NACIONES UnidAS (1992). Declaración final. Conferencia de las Naciones Unidas sobre el Medio Ambiente y el Desarrollo, 3-14 de junio. Río de Janeiro. A/ CONF. 151/26 (vol. I) Anexo I. Descargado el 5 de septiembre de 2013. http://www. un.org/esa/dsd/agenda21_spanish/res_riodecl.shtml. También en: Agenda 21. Cap. 36. http://www.un.org/esa/dsd/agenda21_spanish/res_agenda21_36.shtml).

ORGANIZACIÓN DE LAS NACIONES UNIDAS (2002). Declaración de Johannesburgo sobre el Desarrollo Sostenible. En Informe de la Cumbre Mundial sobre Desarrollo Sostenible (A/ CONF.199/20). Descargado el 5 de septiembre de 2013. http://archive.unctad.org/sp/ docs/aconf199d20\&c1_sp.pdf.

OrganizACión de las NACIONES Unidas (2012). El futuro que queremos. Declaración Final. Conferencia de las Naciones Unidas sobre Desarrollo Sostenible (Río + 20), 20-22 de junio, Río de Janeiro. A/CONF.216/L.1. Descargado el 5 de septiembre de 2013. https:// rio20.un.org/sites/rio20.un.org/files/a-conf.216-1-1_spanish.pdf.pdf.

Real Decreto 1333/1991 de 6 de septiembre ( $B O E$ día 9) sobre currículo de Educación Infantil.

Tilbury, D. (2011). Education for Sustainable Development. An Expert Review of Processes and Learning. París: Unesco. Descargado el 15 de octubre de 2013. http://unesdoc. unesco.org/images/0019/001914/191442e.pdf. 
Uceda, C. y Fort, M. (2011). La educación Ambiental en el sistema educativo. De los 70 a las A21E. En M. Melendro Estefanía, M. ${ }^{a}$ Á. Murga-Menoyo y A. Cano Pérez. IDEAS. Iniciativas de Educación Ambiental para la Sostenibilidad (pp. 37-50). Madrid: UNED.

UNESCO (2005). Directrices y Recomendaciones encaminadas a reorientar la formación de docentes para abordar el tema de la sostenibilidad. La educación para el desarrollo sostenible en la práctica. Documento técnico n. ${ }^{\circ} 2$. París. Descargado el 13 de septiembre de 2013. http://unesdoc.unesco.org/images/0014/001433/143370s.pdf.

UNESCO (2006). Framework for the UNDESD International Implementation Scheme. ED/ DESD/2006/PI/1. París: UNESCO.

UNESCO (2010). Education for Sustainable Development Lens: A Policy and Practice Review Tool. Education for Sustainable Development in Action. Learning and Training Tools, n. ${ }^{\circ}$ 2. Paris: Section for Education for Sustainable Development (ED/UNP/DESD) UNESCO.

UNESCO (2012a). El fomento de competencias para el desarrollo sostenible. Paris: UNESCO.

unESCO (2012b). Educación para el desarrollo sostenible. Paris: Unesco. Descargado el 13 de septiembre de 2013. http://unesdoc.unesco.org/images/0021/002167/216756s.pdf.

Vázquez, A. y Massanero, M. A. (2005). Actitudes de los jóvenes en relación con los desafíos medio-ambientales. Infancia y Aprendizaje, 28 (3), 309-327. http://dx.doi.org/10.1174/0210370054740269

Vega Marcote, P. y Álvarez Suárez, P. (2006). Planteamiento de un marco teórico de la Educación Ambiental para un desarrollo sostenible. Revista Electrónica de Enseñanza de las Ciencias, vol. 4 (1), s/p. Descargado el 14 de marzo de 2014. http://reec.uvigo.es/ volumenes/volumen4/ART4_Vol4_N1.pdf. 